\title{
Synthesis and Characterization of PVP capped Er and Cu co- doped ZnS Nanoparticles
}

\author{
S. Rajesh ${ }^{1}$, K. Thyagarajan ${ }^{1}$, G.S. Harish ${ }^{2}$, Vasudeva Reddy Minnam Reddy ${ }^{3}$ \\ ${ }^{I}$ (Department of Physics, JNTUA College of Engineering, Pulivendula-516390, Kadapa, India.) \\ ${ }_{2}^{2}$ (Government Polytechnic, Satyavedu-517588, India.) \\ ${ }^{3}$ School of Chemical Engineering, Yeungnam University, 280 Daehak-ro, Gyeongsan 38541, South \\ Korea
}

\begin{abstract}
Erbium and Copper co-doped Zinc Sulfide nanoparticles were synthesized using a soft chemical co-precipitation method at different concentrations of PVP (polyvinylpyrrolidone). The structural, morphological, compositional and photoluminescence properties of PVP capped and uncapped ZnS: Er, Cu nanoparticles were studied using X-ray diffraction (XRD), transmission electron microscope (TEM), energy dispersive analysis of X-rays (EDAX), Raman spectroscopy and photoluminescence (PL) techniques. XRD patterns showed that the PVP capped and uncapped $\mathrm{ZnS}: \mathrm{Er}, \mathrm{Cu}$ nanoparticles have cubic zinc blende structure with the average crystalline sizes of about $4-9 \mathrm{~nm}$. The particle size observed from TEM analysis is in good agreement with XRD studies. The EDAX confirmed the effective doping of Er and Cu into ZnS host lattice. No impurity peaks were observed in the Raman spectra of the prepared samples. The room temperature photoluminescence (PL) spectrum of PVP capped and uncapped $\mathrm{ZnS}: \mathrm{Er}, \mathrm{Cu}$ nanoparticles exhibited luminescence peaks at 540-550 $\mathrm{nm}$. The PL intensity of the prepared samples enhanced with increasing PVP concentration.
\end{abstract}

Keywords: particle size, TEM, Raman spectra, photoluminescence.

\section{Introduction}

In recent years, some unique characteristics of $\mathrm{ZnS}$ nanocrystals different from bulk crystals have enlarged the range of applications. Zinc sulfide $(\mathrm{ZnS})$ as an important wide-band gap $(3.6 \mathrm{eV})$ semiconductor has been used as a key material in ultraviolet light-emitting diodes and injection lasers, flat-panel displays, electroluminescent devices and infrared windows [1,2]. The rapid advancement in nanotechnology and biotechnology, particularly the development of new methods for materials synthesis, force towards the controlled synthesis, optical properties, magnetic properties and bio-applications of rare-earth ions doped materials on the nano-scale. Ln3+-doped inorganic luminescent nanomaterials, emerging as a new class of luminescent bio-probes and as an alternative to conventional molecular probes, have been well developed and pushed forward with unprecedented speed towards diverse biomedical applications in recent years. These nanobioprobes inherit the unique optical properties of $\mathrm{Ln}^{3+}$ ions such as long-lived luminescence, large antennagenerated Stokes or anti-Stokes shifts, narrow emission bands, high resistance to photo-bleaching and low toxicity [3]. It is well known that rare earth (RE) elements are effective luminescent centres for RE-doped semiconductors, because the excitation of the RE ions can occur by the recombination of photo generated carriers confined in the semiconductor, and subsequent energy transfer to the RE ions. In this present study, $\mathrm{ZnS}$ : $\mathrm{Er}, \mathrm{Cu}$ nanoparticles capped with PVP is prepared by chemical co-precipitation technique. In this article we report the synthesis and characterization of transition metal and rare earth metal co-doped $\mathrm{ZnS}$ nanomaterials capped with polyvinylpyrrolidone (PVP). The unique luminescence property such as strong and enhanced visible light emission due to transition metal and rare earth ions co-doped $\mathrm{ZnS}$ nanoparticles and the effect of capping agent namely PVP are discussed.

\section{Experimental Details}

All chemicals were of analytical reagent grade and were used without further purification. The samples were prepared by chemical co-precipitation method using pure zinc acetate, Erbium (III) Chloride, Copper (II) acetate and Sodium sulfide with PVP as capping agent. Appropriate amounts of $\mathrm{Zn}(\mathrm{ac})_{2}, \mathrm{ErCl}_{3} \cdot 6 \mathrm{H}_{2} \mathrm{O}$ and $\mathrm{Cu}(\mathrm{OAc})_{2}$ were dissolved in distilled water. In a typical synthesis, desired molar proportions of $\mathrm{Zn}$ $\left(\mathrm{CH}_{3} \mathrm{COO}\right)_{2} \cdot 2 \mathrm{H}_{2} \mathrm{O}, \mathrm{ErCl}_{3} \cdot 6 \mathrm{H}_{2} \mathrm{O}, \mathrm{Cu}\left(\mathrm{CH}_{3} \mathrm{COO}\right)_{2} \cdot \mathrm{H}_{2} \mathrm{O}$ and $\mathrm{PVP}$ each were dissolved in $50 \mathrm{ml}$ Ultrapure deionized water and stirred for 60 minutes. Later $\mathrm{Na}_{2} \mathrm{~S}$ solution was drop wisely added to the solution at room temperature under constant stirring and Stirring was continued for 3 hours. The obtained precipitate was washed with de-ionized water and ethanol for several times. Finally, the powders were vacuum dried for 3 hours at $80^{\circ} \mathrm{C}$ 
to obtain PVP capped ZnS: Er, Cu nanoparticles. Undoped ZnS nanoparticles were synthesized by the same procedure.

\section{Characterization Techniques}

The X-ray diffraction patterns of the samples were collected on a Rigaku D X-ray diffractometer with the $\mathrm{Cu}-\mathrm{K} \alpha$ radiation $\left(\lambda=1.5406 \mathrm{~A}^{\circ}\right)$. Elemental composition of the prepared samples was analyzed through EDAX using an Oxford Inca Penta FeTX3 EDS instrument attached to Carl Zeiss EVO MA 15 Scanning Electron Microscope. Morphology and particle size were analyzed using a TECHNAI-TEM FEI Transmission Electron Microscope (TEM), operated at an accelerating voltage of 100-200 kV. Photoluminescence spectra were recorded in the wavelength range of 400-650 nm using a PTI (Photon Technology International) Fluorimeter with a Xe-arc lamp of power $60 \mathrm{~W}$ and an excitation wavelength of $320 \mathrm{~nm}$ was used. The Raman Spectroscopic studies of the as prepared samples were carried out using a LabRam HR800 Raman Spectrometer.

\subsection{Structural Analysis}

\section{Results And Discussions}

Fig. 1 represents the XRD patterns of uncapped Er, Cu co-doped ZnS nanoparticles and PVP capped $\mathrm{ZnS}$ : Er, Cu nanoparticles. All the uncapped and PVP capped samples are well-crystallized, and the diffraction peaks can be indexed to cubic (fcc) ZnS (JCPDS card no. 80-0020). XRD studies (shown in Fig. 1) revealed cubic zinc blende structure and Dopant incorporation was confirmed by lattice contraction and nanocrystalline nature with broadened XRD pattern which is consistent with TEM studies.

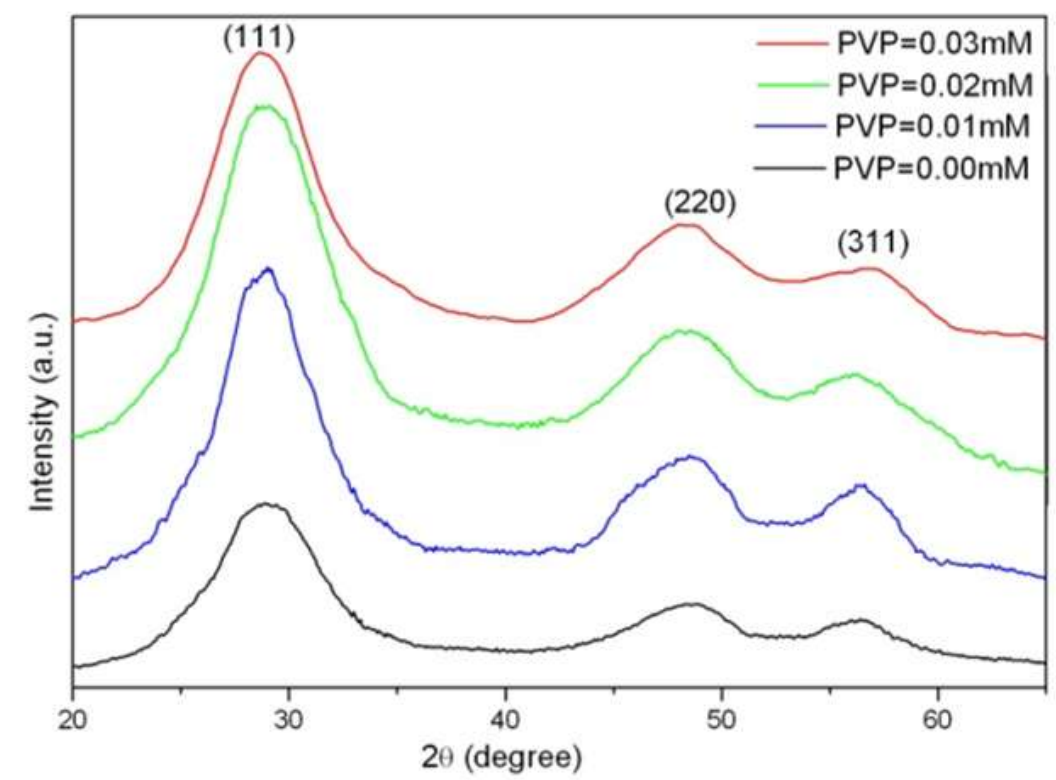

Fig. 1 XRD profiles of PVP capped Er, Cu co-doped ZnS nanoparticles at different PVP concentrations

The average particle size of the samples is calculated by Debye Scherrer's equation (1) and tabulated in Table1.

$$
D=\frac{0.94 \lambda}{\beta_{h k l} \cos \theta}
$$

Where, $\mathrm{D}$ is the average particle size and $\beta_{\mathrm{hkl}}$ is full width at half maximum of XRD peak expressed in radians and $\theta$ is the position of the diffraction peak.

TABLE 1. Average Particle size of PVP capped Er, Cu co-doped $\mathrm{ZnS}$ nanoparticles.

\begin{tabular}{|c|c|c|}
\hline S.No & PVP concentration(M) & Particle size(nm) \\
\hline 1 & $0.00 \mathrm{mM}$ & 9 \\
\hline 2 & $0.01 \mathrm{mM}$ & 7 \\
\hline 3 & $0.02 \mathrm{mM}$ & 4 \\
\hline 4 & $0.03 \mathrm{mM}$ & 6 \\
\hline
\end{tabular}

\subsection{Morphological and Compositional Analysis}

The typical TEM micrograph of $\mathrm{ZnS}$ : Er, Cu nanoparticles capped with PVP is shown in Figure 2, which establish the reasonable uniformity of the particle size. The TEM images reveal that the nanocrystals are faceted, crystalline and of average size of $\sim 4-9 \mathrm{~nm}$. 


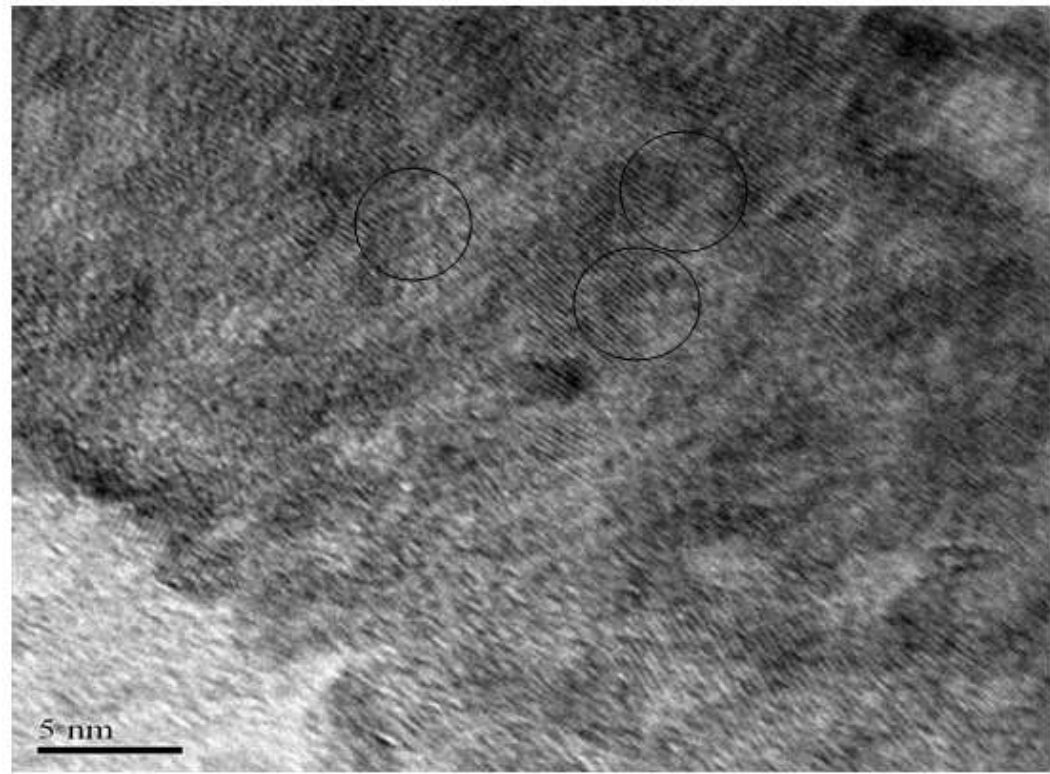

Fig. 1. Typical TEM micrograph of PVP capped Er, Cu co-doped ZnS nanoparticles

The EDS analysis demonstrated that $\mathrm{Zn}, \mathrm{Er}, \mathrm{Cu}$ and $\mathrm{S}$ elements are present in the sample which further confirmed the successful doping of $\mathrm{Er}$ and $\mathrm{Cu}$ in the $\mathrm{ZnS}$ host structure. From Figure 3, it is clear that the chemical compositions of the constituents in the samples, obtained from EDS compatible with starting material compositions. The EDS spectrum confirmed the composition of the elements ( $\mathrm{Zn}, \mathrm{Er}, \mathrm{Cu}$ and $\mathrm{S}$ ) in the sample.

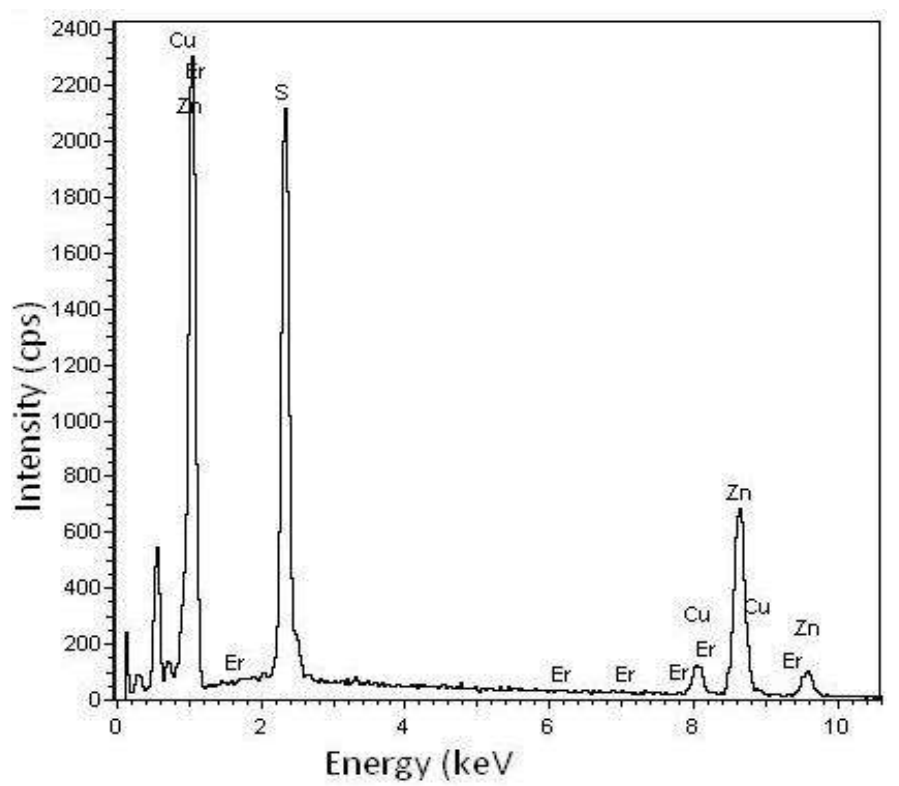

Fig. 3. Typical EDAX spectrum of Er, Cu co-doped $\mathrm{ZnS}$ nanoparticles

\subsection{Photoluminescence and Raman studies}

Figure 4 shows the PL spectra for uncapped and PVP capped ZnS: Er, Cu nanoparticles recorded at room temperature with an excitation wavelength of $320 \mathrm{~nm}$. The emission peaks are observed at 540-550 nm and is well supported by the reports of P. Yang et al.[4]. The PL intensity of the sample co-doped with Er and $\mathrm{Cu}$ is enhanced with increasing the PVP concentration up to $0.02 \mathrm{mM}$ and thereafter, PL intensity was decreased. This decrease in PL intensity could be due to the intra ionic non-radiative relaxation between the adjacent $\mathrm{Er}, \mathrm{Cu}$ ions and PVP. 


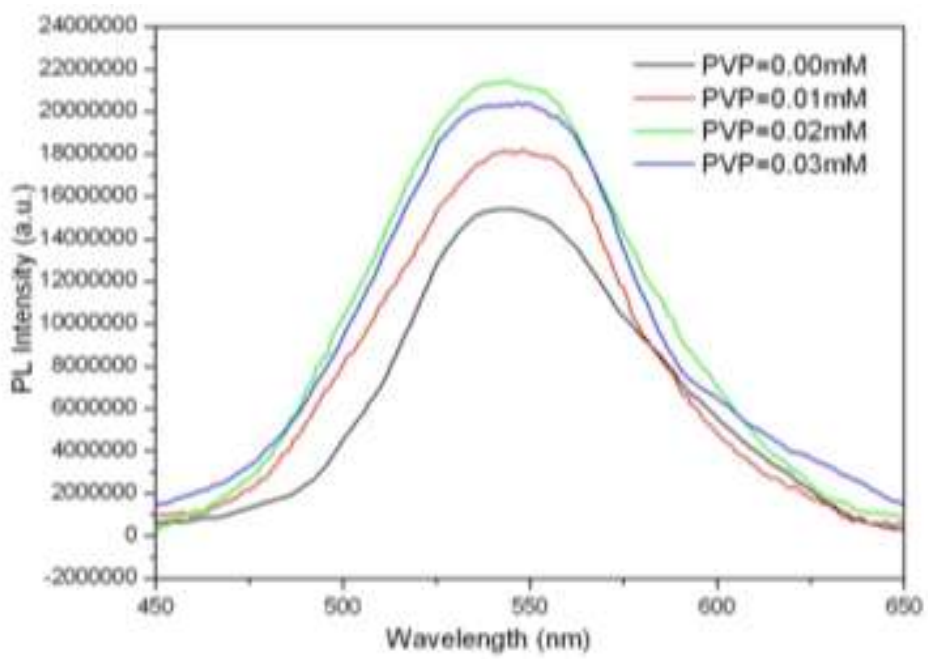

Fig. 4. Photoluminescence spectra of uncapped and PVP capped ZnS: Er, Cu nanoparticles

Raman spectra of the uncapped and PVP capped ZnS: Er, Cu nanoparticles in the frequency range $200-400 \mathrm{~cm}^{-}$ ${ }^{1}$ is shown in Figure 5. The Raman spectrum of prepared samples exhibited strong but broad peaks at 258 and $344 \mathrm{~cm}^{-1}$.

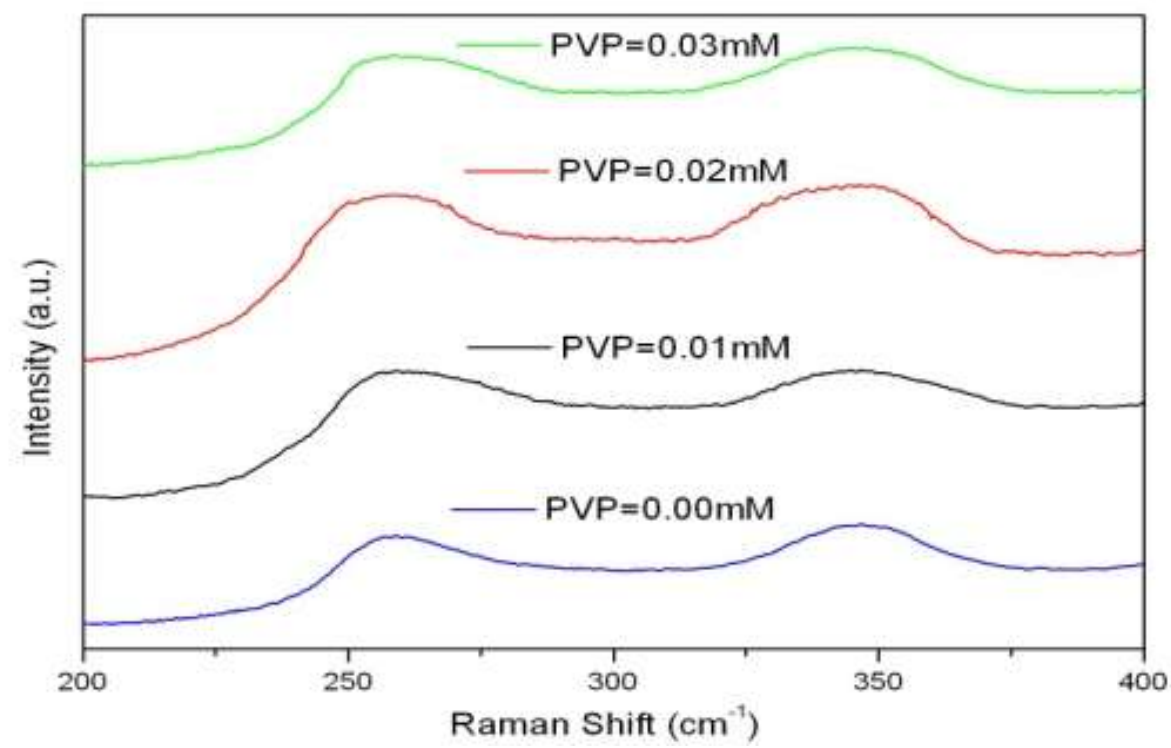

Fig. 5. Raman spectra of uncapped and PVP capped ZnS: Er, Cu nanoparticles

The Raman peak observed at $344 \mathrm{~cm}^{-1}$ can be assigned as the LO mode of cubic ZnS [5]. Brafman et. al. [6] observed the E2 modes of wurtzite type $\mathrm{ZnS}$ at 72 and $286 \mathrm{~cm}^{-1}$. The absence of any peak around 72 and $286 \mathrm{~cm}^{-1}$ in the Raman spectra of the present samples confirmed the phase of the sample to be cubic.

\section{Conclusions}

In summary, ZnS: Er, Cu nanoparticles capped with PVP have been successfully synthesized by chemical co-precipitation method. XRD and Raman spectra confirmed that the prepared nanoparticles have cubic zinc blende structure. EDAX confirmed the effective doping of the corresponding elements and the particle size and distribution were successfully studied by TEM. As the concentration of PVP increases, particle size of the samples decreased and PL intensity enhanced upto PVP= $0.02 \mathrm{mM}$.

\section{Acknowledgments}

The author would like to thank those who have contributed helpful discussions and encouragement, especially Dr. K. Thyagarajan, Department of Physics and sincerely thank the Management of TEQIP-II JNTUCEP, Pulivendula, Andhra Pradesh, India, for providing the necessary amenities for the work. The author S.Rajesh would like to thank Prof. P. Sreedhara Redddy, Department of Physics, S.V. University, Tirupathi, 
India for providing SEM analysis and also Prof. C.K. Jayasankar, [MoU-DAE-BRNS Project (No.2006/34/36/BRNS/3174)], Department of Physics, S.V. University, Tirupathi, India for extending experimental facility.

\section{References}

[1] T. Kushida, A. Kurita, M. Watanabe, Y. Kanematsu, K. Hirata, N. Okubo and Y. Kanemitsu, J. Lumin. 87-89, 466-468 (2000).

[2] R. Sarkar, C.S. Tiwary, P. Kumbhakar, S. Basu and A.K. Mitra, Physica E 40, 3115-3120 (2008).

[3] S. Sambasivama, B. Sathyaseelan, D. Raja Reddy, B.K. Reddy and C.K. Jayasankar, Spectrochim.Acta, Part A 71, 1503-1506 (2008).

[4] P. Yang, M. Lu, D. Xu, D. Yuan and G. Zhou, J. Lumin, 93, 101-105 (2001).

[5] S. Saravana Kumar, M. Abdul Khadar, S.K. Dhara, T.R. Ravindran and K.G.M. Nair, Nucl. Instr and Meth. in Phys. Res. B 251, 435-440(2006).

[6] O. Brafman and S.S. Mitra, Phys. Rev. 171, 931-934 (1968). 